\title{
MRI of the pharynx and treatment efficacy of a mandibular advancement device in obstructive sleep apnoea syndrome
}

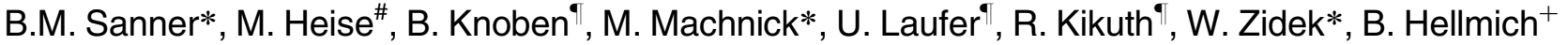

MRI of the pharynx and treatment efficacy of a mandibular advancement device in obstructive sleep apnoea syndrome. B. M. Sanner, M. Heise, B. Knoben, M. Machnick, U. Laufer, R. Kikuth, W. Zidek, B. Hellmich. C ERS Journals Ltd 2002.

ABSTRACT: In obstructive sleep apnoea syndrome (OSAS), prosthetic mandibular advancement devices (MAD) seem to be a promising treatment alternative to conventional continuous positive airway pressure therapy. Unfortunately, while they are effective in some patients, they are ineffective in others or may even worsen OSAS. At present, it is not known whether predictors can be defined which allow for estimation of the potential effect of oral appliances on the severity of OSAS.

Clinical and polysomnographical efficacy of a MAD was evaluated in 15 patients with OSAS. In addition, ultrafast magnetic resonance imaging (MRI) of the pharynx was performed in 13 of these patients at rest during transnasal shallow respiration and during performance of the Muller manoeuvre, both with and without the MAD, and the site of closure was determined.

The MAD reduced the mean apnoea/hypopnoea index (AHI) from $19.8 \pm 14.5$ to $7.2 \pm 7.4 \cdot h^{-1}$. Seven subjects $(53.8 \%)$ had at least a $50 \%$ reduction in AHI to a value $<\mathbf{1 0} \cdot \mathrm{h}^{-1}$ with the MAD, whereas the MAD was ineffective in six patients. Five of the seven treatment responders had no significant pharyngeal obstruction during the manoeuvre with the device, while all of them had pharyngeal obstruction when not equipped with the device. Four of the six patients with treatment failure had a single velopharyngeal obstruction and two a combined obstruction of the velo- and glossopharynx during the Muller manoeuvre while wearing the device.

The results of this study suggest that airway patency during the Muller manoeuvre while wearing a mandibular advancement device may be predictive of the success of obstructive sleep apnoea syndrome treatment with a mandibular advancement device. Eur Respir J 2002; 20: 143-150.
*Dept of Medicine I, Ruhr University Bochum, Marienhospital Herne, ${ }^{\#}$ Dept of Orthodontics, Witten University, 'Dept of Radiology, Ruhr University Bochum, Marienhospital Herne and Dept of Medicine, Ruhr University Bochum, Universitaetsklinik Bergmannsheil, Germany.

Correspondence: B.M. Sanner, Medizinische Klinik, Bethesda Krankenhaus Wuppertal $\mathrm{GmbH}$, Akademisches Lehrkrankenhaus der Ruhr-Universität Bochum, Hainstr. 35, 42109 Wuppertal, Germany.

Fax: 492022902005

E-mail: Bernd.Sanner@.

ruhr-uni-bochum.de

\section{Keywords: Magnetic resonance}

imaging

mandibular position

obstructive sleep apnoea

oral appliance

Received: August 32001

Accepted after revision: February 18 2002
Nasal continuous positive airway pressure (CPAP) is well established, widely used, and effective in the treatment of obstructive sleep apnoea syndrome (OSAS) [1]. It has been shown that CPAP not only improves daytime function but also quality of life [2]. Unfortunately, side-effects are frequent during CPAP treatment, as $>50 \%$ of patients complain of problems due to the nasal mask or of symptoms such as nasal congestion, dry nose, mouth or throat, and discomfort associated with cold air [3-5]. These symptoms may compromise a patient's ability to successfully utilise CPAP and impair compliance with this treatment [6]. Compliance may vary depending on technical and medical follow-up, with previous studies reporting levels as high as $80 \%$ after 3 months of treatment [7] but also as low as $46 \%$ [8].

Poor compliance is the reason for the increased use of alternative treatment modalities, especially of oral appliances. Oral appliances appear to be a promising treatment since they are noninvasive, reversible, quiet and readily accepted by the patients [9]. Unfortunately, while they are effective in some patients, they are ineffective in others or may even worsen OSAS. At present, it is not known whether predictors can be defined which allow the estimation of the potential effect of oral appliances on the severity of OSAS.

For this purpose, a prospective study in patients with OSAS who were treated with a mandibular advancement device (MAD) with an adjustable hinge that allows progressive advancement of the mandible was conducted, and magnetic resonance imaging (MRI) was used to document the effects of the device on anatomical structures. It was hypothesised that airway patency during the Muller manoeuvre would predict the outcome of MAD treatment in patients with OSAS.

\section{Materials and methods}

Fifteen consecutive patients to whom either treatment with CPAP was not suggested because of mild OSAS, or who rejected CPAP, were invited to participate in the study.

The inclusion criterion was polysomnographically verified OSAS with an apnoea/hypopnoea index (AHI) of at least $5 \cdot \mathrm{h}^{-1}$ of sleep. Exclusion criteria included edentulous jaws, pain from the craniomandibular 
system, weight $>300$ pounds (the MRI scanner table limit), as well as the usual contraindications to MRI scanning (e.g. ferromagnetic clips) or age $<18$ or $>75$ yrs. No patient was receiving sedatives or muscle relaxants and there were no shift workers in the population.

The study protocol was approved by the Ethics Committee of the University of Bochum. Informed consent was obtained from all patients before entry into the study.

\section{Study design}

OSAS was diagnosed by polysomnography, and daytime sleepiness was assessed by the Epworth sleepiness scale [10]. Patients who agreed to participate in the study were supplied with a MAD. Four weeks later, to allow adaptation to the device, patients were readmitted in order to repeat the daytime assessment of sleepiness and the efficacy and side-effects of the MAD. They were evaluated with a questionnaire. In addition, MRI scanning was performed with and without the device. On the following night, polysomnography was performed with the device in place, in order to objectively document the influence of treatment on the severity of OSAS. Treatment success with the device was defined if the AHI had sunk by at least $50 \%$ to a value $<10 \cdot \mathrm{h}^{-1}$ of sleep.

\section{Polysomnography}

All patients underwent overnight polysomnography (Somnostar 4100; SensorMedics Co., Yorba Linda, CA, USA) according to widely accepted methods [11]. The transducers and leads permitted normal positional changes during sleep. Bedtime and awakening time were at each subject's discretion. Polysomnography was terminated after final waking. The entire recording was supervised by a technician.

According to the commonly used clinical criteria [12], polysomnography records were scored in 30-s periods for sleep, breathing, and oxygenation. The average number of episodes of apnoea and hypopnoea per hour of sleep (AHI) was calculated. OSAS was diagnosed when the AHI was $>5 \cdot \mathrm{h}^{-1}$, associated with typical clinical features. Sleep was staged manually using the methods of RECHTSCHAFFEN and KALES [13].

\section{Mandibular advancement device}

After the diagnostic polysomnogram the MAD was fitted individually to all patients. After impressions of the upper and the lower jaws were taken, super hard plaster diagnostic casts were fabricated in the laboratory for individual occlusion of the jaws (Dr. Hinz Laboratory, Herne, Germany). The maximum protrusion of the lower jaw was determined in each individual and a George-Gauge ${ }^{\mathrm{TM}}$ (H Orthodontics, Michigan City, IN, USA) registration was taken with $65 \%$ of the maximum anterior movement. This position was fixed by a bite-registration silicone (3M, Neuss, Germany). Thus, each patient was allowed an individual protrusion as well as a downward rotation of the mandible, leading to an increase of both the vertical and the lateral dimension of the jaw.

The upper and lower casts were mounted on an articulator via the registration with the GeorgeGauge $^{\mathrm{TM}}$. The removable occlusal splints consisted of translucent polymethane polycarbonate (Durasoft $2.5 \mathrm{~mm}$; Scheu Dental, Iserlohn, Germany). The device was retained on the teeth by friction fit of thermoplastic in undercuts. For periodontal prophylaxis there was a minimum distance of $1 \mathrm{~mm}$ to the gingiva. Afterwards the vertical dimension stops were polymerised from acrylic for rotating the mandible downwards with an open frontal space to assure adequate respiration.

The smooth adjustable stainless-steel protrusion sticks allowed progressive advancement of the mandible. They were polymerised on the buccal side of the dorsal ends of teeth 16 and 26, and on the anterior side of teeth 34 and 44. Thus, an irritation of the stomions was avoided. In addition, the two-piece mandibular advancers could be adjusted easily to increase the mandibular advancement. In this study group, the mandible was advanced to $65 \%$ of maximum.

To aid initial habituation to the foreign body the patient was instructed to insert the device $1-2 \mathrm{~h}$ prior to bedtime and then to wear it throughout the night.

\section{Magnetic resonance imaging}

A 1.5 Tesla magnetic resonance scanner (Signa; General Electric, Milwaukee, WI, USA) with a standard head coil was used. The pharyngeal airway was imaged by ultra-fast two-dimensional sequences in midsagittal and axial projection during transnasal shallow respiration at rest and during performance of the Muller manoeuvre, allowing visualisation of the position of the tongue, soft palate, uvula and posterior pharyngeal surface. The measurements were performed in awake patients in the supine position with the heads in a neutral anatomical position with a T1 weighted FSPGR, a TR of $8.8 \mathrm{~ms}$, a TE of $2 \mathrm{~ms}$, a flip angle of $8^{\circ}$, a $300 \times 150 \mathrm{~mm}$ field of view, a $256 \times 128$ acquisition matrix and $5 \mathrm{~mm}$ slice thickness. Eighteen consecutive images were obtained in $30 \mathrm{~s}$.

The Muller manoeuvre consisted of an inspiratory effort with the mouth and nose closed. The nose was closed externally with a clamp. The respiratory manoeuvre, which was initiated after normal inspiration produced a vacuum, which resulted in total or partial collapse of the pharyngeal surface. Patients were asked to continue the Muller manouevre for $30 \mathrm{~s}$. In a second step the measurements were repeated after insertion of the MAD. Obstruction site, length of the obstruction and influence of the Muller manoeuvre and the MAD were analysed. Two observers (BK and UL) measured airway and soft tissue structure dimensions, after previous selection of the most appropriate images, where different structures were clearly visible. A workstation with image processing software (Sun-Spaces 20; Sun Microprocessor, Milwaukee, WI, USA) was used for MRI measurements. 
The part of the nasopharynx where the velum palatinum is located was identified as the velopharynx. The glossopharynx is delimited along its anterior margin by the line of circumvallate papillae of the tongue. It includes the posterior third of the tongue, the palatine, the soft palate, the oropharyngeal mucosa and the top of the epiglottis. "Obstruction" of the velopharynx or glossopharynx was only determined when complete closure occurred. The readers of the MRI scans were blinded to the outcome of MAD treatment.

An attempt was made not to change head position while inserting the oral appliance. To make sure that there was no neck flexion or extension the mid-sagittal scout files were repeated. Patients were instructed to avoid caffeine-containing food or beverages shortly before MRI. They were not allowed to ingest alcohol or sedatives the day before the procedure.

\section{Statistical analyses}

Results are presented as means \pm SD. All p-values reported are two-tailed. Intergroup differences were analysed with the Mann-Whitney test for paired samples, with Bonferroni's correction for multiple comparisons. The relationships between the longitudinal extension of the obstruction on MRI with age, body mass index or the severity of OSAS were explored by bivariate regression analysis. Fisher's exact test was used to compare categorical variables.

\section{Results}

The mean age of the 15 patients (14 males, one female) participating in the study was 57.2 \pm 8.9 yrs (range 35.2-71.4 yrs) and the mean body mass index was $31.4 \pm 5.8 \mathrm{~kg} \cdot \mathrm{m}^{-2}$ (range $24.7-46.4 \mathrm{~kg} \cdot \mathrm{m}^{-2}$ ).

\section{Clinical results with the oral device}

All patients tolerated the MAD well and reported daily use of the appliance. Dental side-effects were frequent, although these were mostly of a minor and temporary nature and none of the patients ceased using the device because of them. The subjective

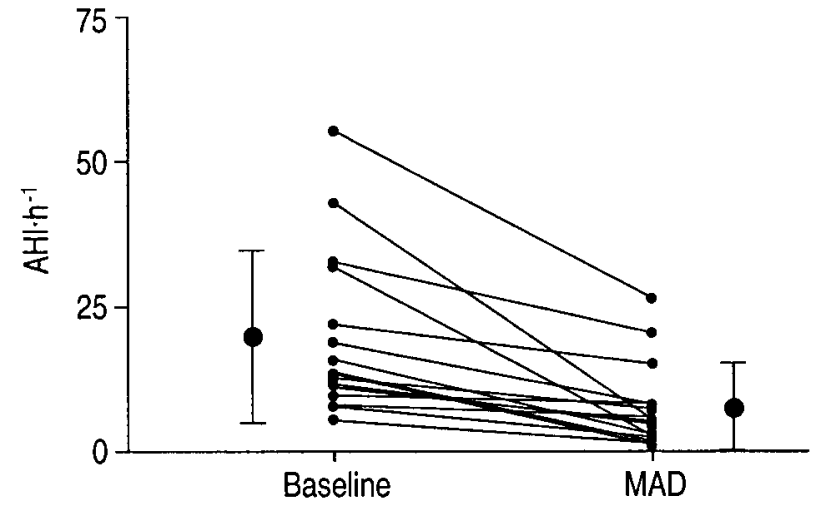

Fig. 1.-Apnoea/hypopnoea index (AHI) without and with the mandibular advancement device (MAD).

benefit was large enough to overcome the discomfort. Side-effects included dental discomfort (11 patients), xerostomia (nine patients), excess salivation (eight patients), bite change (seven patients) and temporomandibular joint pain (five patients). The MAD did not cause mouth opening.

Treatment with the MAD improved daytime sleepiness. Epworth sleepiness scale decreased from $10.1 \pm 5.6$ to $7.2 \pm 4.0(\mathrm{p}<0.05)$.

\section{Polysomnographic results with and without the mandibular advancement device}

For the entire group, MAD reduced the AHI from $19.8 \pm 14.5 \mathrm{~h}$ of sleep $^{-1}$ (range $5.4-55.2 \mathrm{~h}^{-1}$ sleep $^{-1}$ ) to $7.2 \pm 7.4 \mathrm{~h}$ of sleep $^{-1}$ (range $0.3-25.9 \mathrm{~h}$ of sleep ${ }^{-1}$ ) $(\mathrm{p}=0.001)$. The individual values are given in figure 1. There were no significant differences in sleep architecture with and without the device (table 1). Seven subjects $(53.8 \%$ ) had at least a $50 \%$ reduction in AHI to a value $<10 \cdot \mathrm{h}^{-1}$ with the device (treatment responders), whereas it was ineffective in six patients $(46.2 \%)$ (treatment nonresponders).

Treatment responders and nonresponders did not differ in terms of age, body mass index, value of the initial Epworth sleepiness scale or baseline polysomnographic data.

Table 1.-Effect of therapy with the mandibular advancement device (MAD) on polysomnographic data

\begin{tabular}{lccc}
\hline & Without MAD & With MAD & p-values \\
\hline Total sleep time min & $381.3 \pm 51.0$ & $373.9 \pm 33.5$ & NS \\
Sleep efficiency \% & $92.7 \pm 6.6$ & $92.2 \pm 4.9$ & NS \\
Stage wake \% & $4.4 \pm 4.6$ & $3.4 \pm 3.2$ & NS \\
Sleep stage 1 and 2\% & $62.6 \pm 16.5$ & $67.8 \pm 12.8$ & NS \\
Slow-wave sleep \% & $18.7 \pm 8.1$ & $15.5 \pm 8.0$ & NS \\
Sleep stage REM \% & $10.8 \pm 7.6$ & $12.8 \pm 6.8$ & NS \\
Apnoea/hypopnoea index $\mathrm{n} \cdot \mathrm{h}^{-1}$ & $19.8 \pm 14.5$ & $7.2 \pm 7.4$ & $=0.01$ \\
Arousal index $\mathrm{n} \cdot \mathrm{h}^{-1}$ & $19.3 \pm 9.9$ & $10.4 \pm 6.8$ & $<0.05$ \\
Minimum nocturnal $\mathrm{Sa}_{\mathrm{a}} \%$ & $85.4 \pm 6.8$ & $\mathrm{NS}$ \\
Mean nocturnal $\mathrm{S}_{\mathrm{a}, \mathrm{O}_{2} \%} \%$ & $94.8 \pm 1.9$ & $\mathrm{NS}$ \\
\hline
\end{tabular}

Data are presented as means \pm SD. REM: rapid eye movement; $\mathrm{Sa}_{\mathrm{a}} \mathrm{O}_{2}$ : arterial oxygen saturation. 


\section{Magnetic resonance imaging}

With the MRI protocol, dynamic imaging of the pharynx was performed completely in 13 patients with good air to soft tissue contrast without the use of any paramagnetic contrast medium. Two patients did not complete the examination because of claustrophobia.

Two of the 13 patients had velopharyngeal obstruction during shallow breathing at rest. During the Muller manoeuvre, pharyngeal obstruction could be observed in all patients: five patients had a single obstruction of the velopharynx, four patients a single obstruction of the glossopharynx and four patients a combined obstruction of the velopharynx and glossopharynx. The mean longitudinal extension of

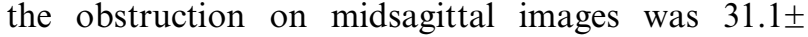
$24.8 \mathrm{~mm}$. Obstructions of the velo- and glossopharynx, as detected on sagittal projections, correlated well with the same occlusions detected on axial projections.

There was no correlation between the longitudinal extension of the obstruction and age, body mass index, or the severity of OSAS.

Repeat MRI was performed with the dental device. It showed that velopharyngeal obstruction during shallow breathing at rest persisted in the two patients who had the obstruction even without the device. During the Muller manoeuvre performed when equipped with the device, combined obstruction of the glossopharynx and velopharynx was reversed in two cases and single obstruction of the glossopharynx was reversed in three cases compared to the measurements without the device. Combined obstruction could be demonstrated in two patients, single obstruction of the velopharynx in five patients, and single obstruction of the glossopharynx in one patient.

\section{Effect of the site of airway closure on the efficacy of the mandibular advancement device}

In order to find out whether airway patency during the Muller manoeuvre predicted outcome of MAD treatment, a subanalysis was performed. In the seven patients classified as effectively treated with the device (treatment responders), MRI revealed single obstruction of the velopharynx in two, single obstruction of the glossopharynx in three and combined obstruction of the velopharynx and glossopharynx in two patients during the Muller manoeuvre when performed without the device. When equipped with the dental device, obstruction disappeared in the patients with single obstruction of the glossopharynx (fig. 2) and with combined obstruction of the velopharynx and glossopharynx, whereas it persisted in the two patients with single obstruction of the velopharynx.

In six patients, treatment with the dental appliance was ineffective. Two of them had velopharyngeal obstruction during shallow breathing at rest, both with and without the device. During the Muller manoeuvre single obstruction of the velopharynx was evident in four patients and combined obstruction of the velopharynx and glossopharynx in two patients when performed without the device. When equipped with the dental device, obstruction persisted in all patients: single obstruction of the velopharynx could be documented in four patients (fig. 3), and combined obstruction of the velopharynx and glossopharynx in two patients.

All five patients with patent airways during the Muller manoeuvre were responders to the treatment, and six of eight patients with persistent airway occlusion during the Muller manoeuvre were nonresponders $(\mathrm{p}<0.05)$.

\section{Discussion}

The results of this study indicate that the MAD examined in this study is effective in $\sim 50 \%$ of patients and that MRI may help predict clinical and polysomnographic efficacy. Patent airways during the Muller manoeuvre while wearing the device may therefore predict the outcome of MAD treatment in patients with OSAS.

Oral appliances represent an interesting new approach to the treatment of OSAS [9]. Generally, they are divided into two groups, the tongue-retaining device and the MAD. The proposed mechanism of action of the tongue-retaining device is that it holds the tongue in a forward position preventing it from being drawn downward by the negative pressure of inspiration. The other common form is the MAD, as used in this study, which is believed to act by advancing the mandible and increasing upper airway size.

MADs worn intraorally at night have recently been shown in controlled trials to be beneficial in mild to moderate OSAS. Unfortunately, treatment success is only $\sim 50 \%$ [14], a percentage that was also realised with the device used in this study. At present, both the reason for this low success rate and useful predictive parameters of treatment success are not known [15]. It can be speculated that some specific anatomical configuration can be used to predict success or failure of the device [16]. One recently published study measured respiratory fluctuations in oropharyngeal pressure during induced occlusions in nonrapid eye movement sleep [17]. In this study the site of airway closure above or below the oropharynx was determined by the respective presence or absence of respiratory fluctuations. Henke et al. [17] showed that airway closure in the hypopharynx predicted a very favourable outcome. Nonetheless, many patients with velopharyngeal closure, as assumed by oropharyngeal pressure curves, also had improvements in apnoea/hypopnoea indices.

Direct anatomical visualisation of the pharynx in patients with OSAS can be performed with CT and MRI [18, 19], and ultrafast MRI in particular appears to be promising [20].

MRI has been used to characterise changes in the upper airway structure and musculature of patients with OSAS. It was shown that the changes observed in patients with OSAS are compatible with the hypothesis that there is increased oedema and possibly increased fat content of the tongue muscles [21]. 


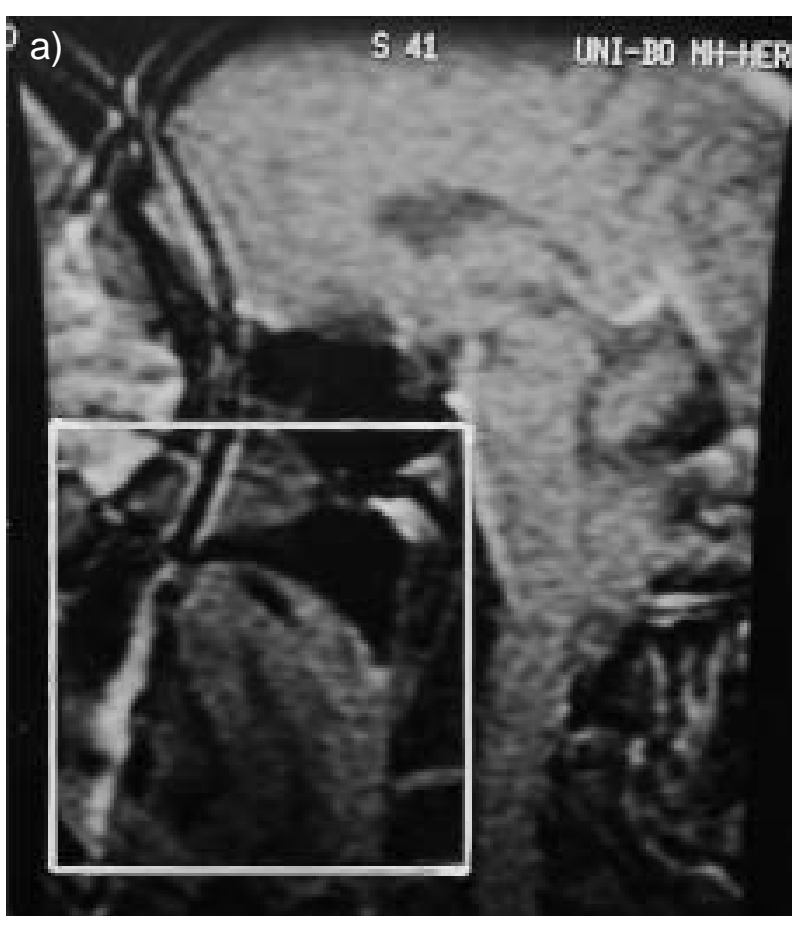

b)
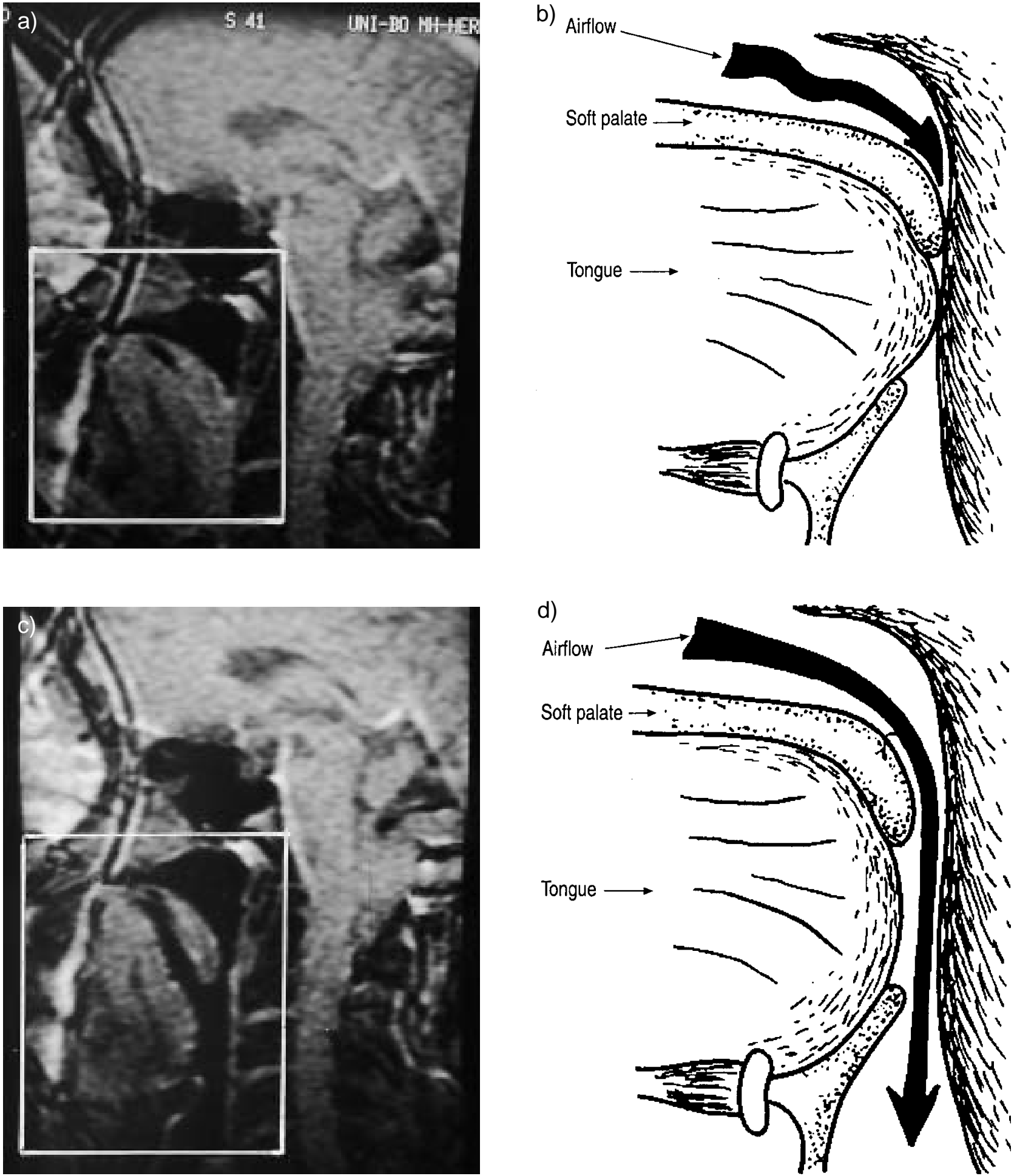

Fig. 2.-Midsagittal magnetic resonance anatomy in a patient performing the Muller manoeuvre a) without and c) with the oral device. The relevant soft tissue structures are highlighted in the schematic drawings b) and d). An obstruction of the glossopharynx is clearly visible without the device. The patient was treated effectively with the device. The upper airway obstruction remained patent with the dental device even during performance of the Muller manoeuvre.

Patients with OSAS have more fat in the areas surrounding the collapsible segment of the pharynx compared to equally obese control subjects without OSAS [22]. Furthermore, the apnoeic airway is smaller and narrowed laterally [23]. The thickness of the lateral pharyngeal muscular walls rather than the enlargement of the parapharyngeal fat pads is the predominant anatomical factor causing airway narrowing in apnoeic subjects. In addition, patients with OSAS have an increased mobility of soft tissue 

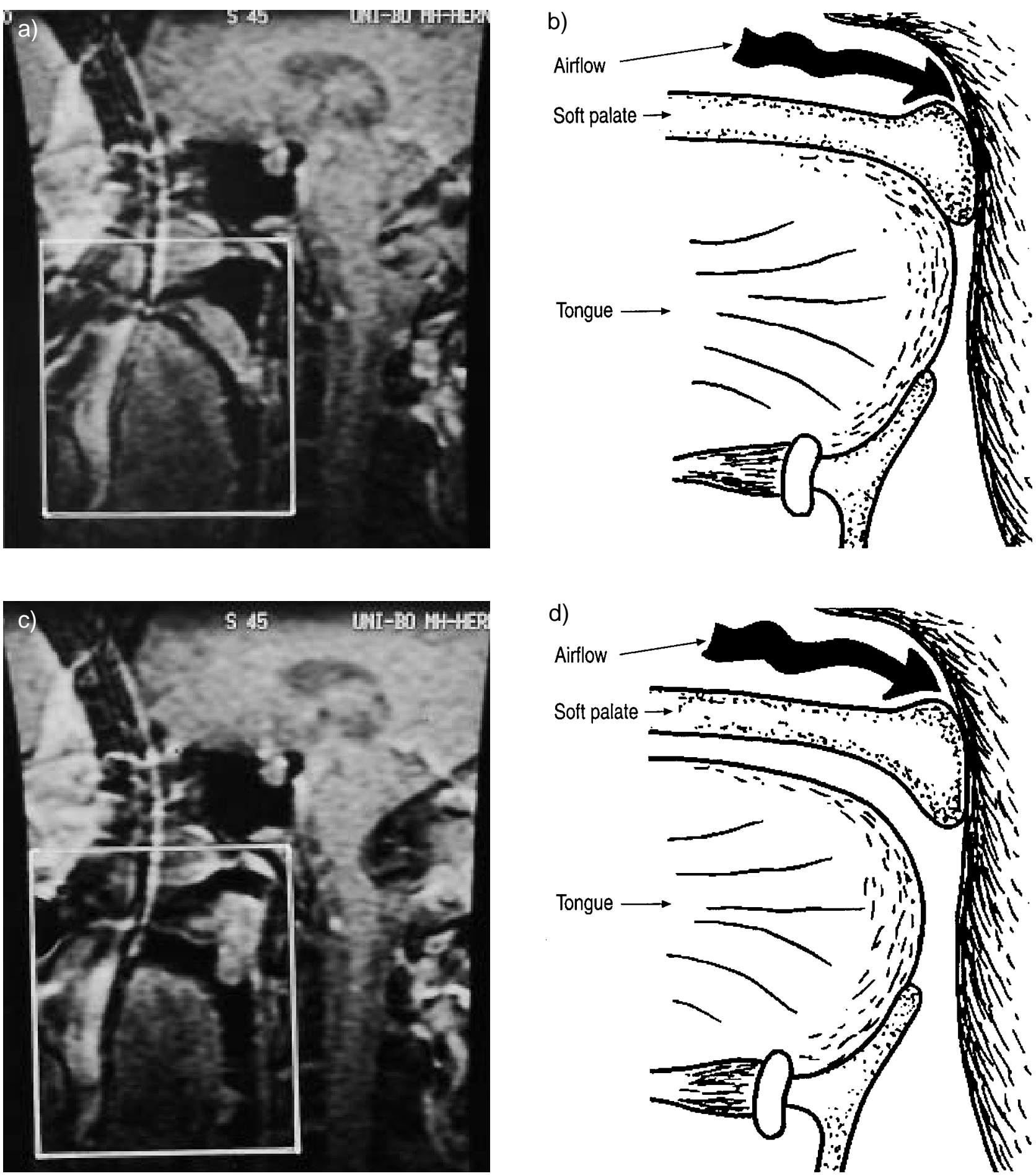

Fig. 3.-Midsagittal magnetic resonance anatomy in a patient performing the Muller manoeuvre a) without and c) with the device. The relevant soft tissue structures are highlighted in the schematic drawings b) and d). An obstruction of the velopharynx is clearly visible, both without and with the device. Polysomnography showed ineffective treatment.

in the velo- and glossopharynx, which results in recurrent obstruction of the pharyngeal airway. Ultrafast MRI performed during sleep has shown that changes in the velopharynx area and diameter during the respiratory cycle are greater in apnoeic patients than in normal subjects, particularly during sleep [24].
In this study the Muller manoeuvre was used to simulate OSAS. In a recently published study using the Muller manoeuvre, it was shown that there are highly significant differences between patients with OSAS and healthy subjects in terms of the degree of obstruction in the velopharynx and glossopharynx [20]. During the Muller manoeuvre pharyngeal 
obstruction could be observed in all of the patients in this study, either a single obstruction of either the velopharynx or the glossopharynx, or a combined obstruction of the velopharynx and glossopharynx.

Use of the MAD changed the anatomical configuration of the upper airway. Five of the seven treatment responders had no significant pharyngeal obstruction during the manoeuvre when equipped with the device while all of them had pharyngeal obstruction without the MAD.

In contrast, two of the six patients with treatment failure had velopharyngeal obstruction even during shallow breathing at rest, both with and without the device, while a single velopharyngeal obstruction was evident in four and a combined obstruction of the velopharynx and the glossopharynx in two patients during the Muller manoeuvre while wearing the device.

The authors are well aware of the limitations of this study: MRI was performed while the patients were awake and this situation probably does not reflect the anatomical abnormality during sleep. Furthermore, the success of the MAD cannot be accurately predicted from anatomical considerations alone. The Muller manoeuvre was used to simulate OSAS, as it is easy to perform, but it does not induce the same negative intrathoracic pressure in all patients and may vary even within an individual. Since the usefulness of the Muller manoeuvre for determination of the site of pharyngeal obstruction is questionable, one has to be very careful about generalisation of the study results, especially in view of the small sample size. By the definition in this study, only complete closure of velopharynx or glossopharynx was used to determine "pharyngeal obstruction". This resulted in a wide variation of "nonobstruction" from wide opening to severe narrowing. Therefore, prospective studies during sleep or with simultaneous recording of intraoesophageal pressures should be performed.

One recent study showed that improvement of both nocturnal oxygenation and pharyngeal collapsibility depends significantly on the mandibular position [25]. In this study, the mandible was advanced to $65 \%$ of maximum in all subjects. This may have lowered the success rate of the MAD.

In conclusion, this study showed that treatment with the mandibular advancement device tested had a significant effect in many, but not all patients with mild to moderate obstructive sleep apnoea syndrome. Furthermore, magnetic resonance imaging may prove to be a useful method for predicting the efficacy of mandibular advancement devices. Airway patency during the Muller manoeuvre while wearing the device may predict outcome of mandibular advancement device treatment in patients with obstructive sleep apnoea syndrome.

\section{References}

1. Sullivan CE, Issa FG, Berthon-Jones M, Eves L. Reversal of obstructive sleep apnea by continuous positive airway pressure through the nares. Lancet 1981; 1: 862-865.
2. Jenkinson C, Davies RJO, Mullins R, Stradling JR. Comparison of therapeutic and subtherapeutic nasal continuous positive airway pressure for obstructive sleep apnoea: a randomized prospective parallel trial. Lancet 1999; 353: 2100-2105.

3. Pepin JL, Leger P, Veale D, Langevin B, Robert D, Levy P. Side effects of nasal continuous positive airway pressure in sleep apnea syndrome. Chest 1995; 107: 375-381.

4. Sanders MH, Gruendl CA, Rogers RM. Patient compliance with nasal CPAP therapy for sleep apnea. Chest 1986; 90: 330-333.

5. Engleman HM, Asgari-Jirhandeh N, McLeod AL, Ramsay CF, Deary IJ, Douglas NJ. Self-reported use of CPAP and benefits of CPAP therapy. Chest 1996; 109: 1470-1476.

6. Redline S, Adams N, Strauss ME, Roebuck T, Winters M, Rosenberg C. Improvement of mild sleep-disordered breathing with CPAP compared with conservative therapy. Am $J$ Respir Crit Care Med 1998; 157: 858-865.

7. Pepin JL, Krieger J, Rodenstein D, et al. Effective compliance during the first 3 months of continuous positive airway pressure. A European prospective study of 121 patients. Am J Respir Crit Care Med 1999; 160: 1124-1129.

8. Kribbs NB, Pack AI, Kline LR, et al. Objective measurements of patterns of nasal CPAP use by patients with obstructive sleep apnea. Am Rev Respir Dis 1993; 147: 887-895.

9. Schmidt-Nowara W, Lowe A, Wiegand L, Cartwright R, Perez-Guerra F, Menn S. Oral appliances for the treatment of snoring and obstructive sleep apnea: a review. Sleep 1995; 18: 501-510.

10. Johns WM. Daytime sleepiness, snoring, and obstructive sleep apnea: the Epworth sleepiness scale. Chest 1993; 103: 30-36.

11. American Thoracic Society-Medical Section of the American Lung Association. Indications and standards for cardiopulmonary sleep studies. Am Rev Respir Dis 1989; 139: 559-568.

12. American Academy of Sleep Medicine Task Force. Sleep-related breathing disorders in adults: Recommendations for syndrome definition and measurement techniques in clinical research. Sleep 1999; 22: 667689.

13. Rechtschaffen A, Kales A. A manual of standardized terminology, techniques and scoring system for sleep stages of human subjects. NIH publication No. 204. Washington, DC, USA. Government Printing Office, 1968.

14. Ferguson KA, Ono T, Lowe A, Al-Majed S, Love L, Fleetham JA. A short term controlled trial of an adjustable oral appliance for the treatment of mild to moderate obstructive sleep apnea. Thorax 1997; 52: 362-368.

15. Schoenhofer B, Stoohs RA, Rager H, Wenzel M, Wenzel G, Köhler D. A new tongue advancement technique for sleep-disordered breathing. Am J Respir Crit Care Med 1997; 155: 732-738.

16. Clark GT, Blumenfeld I, Yoffe N, Peled E, Lavie P. A crossover study comparing the efficacy of continuous positive airway pressure with anterior mandibular positioning devices on patients with obstructive sleep apnea. Chest 1996; 109: 1477-1481.

17. Henke KG, Frantz DE, Kuna ST. An oral elastic 
mandibular advancement device for obstructive sleep apnea. Am J Respir Crit Care Med 2000; 161: 420-425.

18. Galvin JR, Rooholamini SA, Stanford W. Obstructive sleep apnea: diagnosis with ultrafast CT. Radiology 1989; 171: 775-778.

19. Ryan CF, Lowe AA, Li D, Fleetham JA. Magnetic resonance imaging of the upper airway in obstructive sleep apnea before and after chronic nasal continuous airway pressure therapy. Am Rev Respir Dis 1991; 144: 939-944.

20. Jaeger L, Guenther E, Gauger J, Reiser M. Fluoroscopic MR of the pharynx in patients with obstructive sleep apnea. Am J Neuroradiol 1998; 19: 1205-1214.

21. Schotland HM, Insko EK, Schwab RJ. Quantitative magnetic resonance imaging demonstrates alterations of the lingual musculature in obstructive sleep apnea. Sleep 1999; 22: 605-613.
22. Horner RL, Mohiaddin RH, Lowell DG, et al. Sites and sizes of fat deposits around the pharynx in obese patients with obstructive sleep apnoea and weight matched controls. Eur Respir J 1989; 2: 613-622.

23. Schwab RJ, Gupta KB, Gefter WB, Metzger LJ, Hoffman EA, Pack AI. Upper airway and soft tissue anatomy in normal subjects and patients with sleepdisordered breathing. Am J Respir Crit Care Med 1995; 152: 1673-1689.

24. Ciscar MA, Juan G, Martinez V, et al. Magnetic resonance imaging of the pharynx in OSA patients and healthy subjects. Eur Respir $J$ 2001; 17: 79-86.

25. Kato J, Isono S, Tanaka A, et al. Dose-dependent effects of mandibular advancement on pharyngeal mechanics and nocturnal oxygenation in patients with sleep-disordered breathing. Chest 2000; 117: 1065-1072. 